\title{
Irreducible Sp-representations and subgroup distortion in the mapping class group
}

\author{
Nathan Broaddus, Benson Farb and Andrew Putman*
}

\begin{abstract}
We prove that various subgroups of the mapping class group $\operatorname{Mod}(\Sigma)$ of a surface $\Sigma$ are at least exponentially distorted. Examples include the Torelli group (answering a question of Hamenstädt), the "point-pushing" and surface braid subgroups, and the Lagrangian subgroup. Our techniques include a method to compute lower bounds on distortion via representation theory and an extension of Johnson theory to arbitrary subgroups of $\mathrm{H}_{1}(\Sigma ; \mathbb{Z})$.
\end{abstract}

Mathematics Subject Classification (2010). 57M99, 57N05, 20F65, $20 \mathrm{~F} 69$.

Keywords. Mapping class group, Torelli group, subgroup distortion, symplectic representation, Johnson homomorphism.

\section{Introduction}

We begin with a basic motivating question. Manifolds $M$ are commonly presented as gluings or (possibly singular) fiberings of simpler manifolds, together with the data specifying the gluing or fibering. In low dimensions, the gluing/fibering data commonly takes the form of an element, or a finite collection of elements, of the mapping class group $\operatorname{Mod}_{g}$, which is the group of homotopy classes of orientationpreserving homeomorphisms of the closed, oriented, genus $g$ surface $\Sigma_{g}$. Examples include Heegaard decompositions of 3-manifolds, monodromies of surface bundles, and monodromies of Lefschetz fibrations.

The topology of the resulting manifold $M$ can often be controlled by requiring that the gluing data lie in various special subgroups $K<\operatorname{Mod}_{g}$. We then have a purely group-theoretic problem: determine whether or not a given element $f \in \operatorname{Mod}_{g}$, given as a product of generators of $\operatorname{Mod}_{g}$ (say a generating set of Dehn twists), lies in $K$. The problem of finding such an algorithm is called solving the generalized word problem for $K$ in $\operatorname{Mod}_{g}$. The generalized word problem is a classical problem in combinatorial group theory; it was introduced and studied by Nielsen and Magnus.

\footnotetext{
${ }^{*}$ The first two authors are supported in part by the NSF.
} 
A basic example is provided by taking $K$ to be the Torelli group $\mathcal{I}_{g}<\operatorname{Mod}_{g}$, that is, the kernel of the natural symplectic representation $\pi: \operatorname{Mod}_{g} \rightarrow \operatorname{Sp}(2 g, \mathbb{Z})$ given by the action of $\operatorname{Mod}_{g}$ on $\mathrm{H}_{1}\left(\Sigma_{g} ; \mathbb{Z}\right)$. In this case, the solution to the generalized word problem is easy: one simply computes the induced linear map $f_{*} \in \operatorname{Sp}(2 g, \mathbb{Z})$ via matrix multiplication and checks whether or not $f_{*}=\mathrm{Id}$. With this in mind, the more refined and useful problem is to actually express a given element of $\mathcal{I}_{g}$, written in generators for $\operatorname{Mod}_{g}$, in terms of generators of the subgroup $\mathcal{I}_{g}$. A standard quantitative measure of the (in)efficiency of doing this is the notion of distortion, which we now explain.

Distortion in groups. Let $\Gamma$ be a finitely generated group endowed with the word metric $\|\cdot\|_{\Gamma}$. Any finitely generated subgroup $K<\Gamma$ comes equipped with its own word metric $\|\cdot\|_{K}$, and it is a basic problem in geometric group theory to understand the geometry of the embedding $K \hookrightarrow \Gamma$, that is, to compare the "intrinsic metric" $\|\cdot\|_{K}$ with the "extrinsic metric" on $K$ given by the restriction of $\|\cdot\|_{\Gamma}$ to $K$. It is clear that there exist constants $C, C^{\prime}$ such that

$$
\|h\|_{\Gamma} \leq C\|h\|_{K}+C^{\prime} \quad \text { for all } h \in K .
$$

However, there may be "shortcuts" in $\Gamma$ between elements of $K$. This can be encoded by a function $\delta: \mathbb{N} \rightarrow \mathbb{N}$, called the distortion of $K$ in $\Gamma$, which is defined to be the smallest function satisfying

$$
\|h\|_{K} \leq \delta\left(\|h\|_{\Gamma}\right) \quad \text { for all } h \in K .
$$

It is easy to see that $\delta$ changes by at most a linear factor if different generating sets are chosen for $\Gamma$ or for $K$; thus the growth type of $\delta$ (e.g. polynomial of degree $d$, exponential, etc.) is independent of these choices ${ }^{1}$. For an introduction to distortion and its basic properties, see [Gro], [Far1].

We note here that for groups $\Gamma$ with solvable word problem, the distortion of $K$ in $\Gamma$ gives a quantitative measure of the efficiency of solving the generalized word problem for $K$ in $\Gamma$. In particular, the generalized word problem for $K$ in $\Gamma$ is solvable if and only if the distortion of $K$ in $\Gamma$ is recursive (see [Far1]). This problem can be unsolvable in simple examples. For instance, Mihailova (see, e.g. [Far1]) found a finitely generated subgroup $K$ in a product $F_{m} \times F_{m}$ of free groups which has an unsolvable generalized word problem, and hence has nonrecursive distortion.

Statement of results. In this paper we give bounds for the distortions of various subgroups of $\operatorname{Mod}_{g}$. Some results in this direction are already known. Stillwell [Sti] used Mihailova's example to find finitely generated subgroups of $\operatorname{Mod}_{g}$ with

\footnotetext{
${ }^{1}$ Some people use terminology differing from ours by a linear factor, so for example what we call "linearly distorted" they call "undistorted".
} 
nonrecursive distortion. In every other case which has been previously investigated, the distortion has turned out to be linear. Examples of linearly distorted subgroups of $\operatorname{Mod}_{g}$ include abelian subgroups [FLM], subgroups corresponding to mapping class groups of subsurfaces [MM] (see [Ham1] for another proof), and convex, cocompact subgroups [FM].

These results led Hamenstädt to pose the problem (see Problem 6 in [Ham2]) of finding subgroups of $\operatorname{Mod}_{g}$ with (recursive) super-linear distortion. In particular, she asked if the Torelli group $\mathcal{I}_{g}$ is linearly distorted in $\operatorname{Mod}_{g}$. Recall that $\mathcal{I}_{g}$ is finitely generated for $g>2$ by a deep theorem of Johnson ([Jo3], Theorem 2). It was conjectured in [Far2] (Problem 3.7 and the discussion following it) that $\mathcal{I}_{g}$ has exponential distortion in $\operatorname{Mod}_{g}$. Our first result confirms the lower bound of this conjecture.

To state our results in their full generality, let $\Sigma_{g, b}^{p}$ be an oriented genus $g$ surface with $b$ boundary components and $p$ punctures (thought of as marked points), and let $\operatorname{Mod}_{g, b}^{p}$ be the group of homotopy classes of homeomorphisms of $\Sigma_{g, b}^{p}$ which fix the punctures and the boundary components pointwise (either $b$ or $p$ will be omitted from our notation if they equal 0 ). When $b \in\{0,1\}$, the Torelli group $\mathcal{I}_{g, b}$ is defined as the kernel of the action of $\operatorname{Mod}_{g, b}$ on $\mathrm{H}_{1}\left(\Sigma_{g, b} ; \mathbb{Z}\right)$.

Theorem 1.1 (Distortion of the Torelli group). For $g \geq 3$, the distortion of $\mathcal{I}_{g}$ (resp. $\left.\mathcal{I}_{g, 1}\right)$ in $\operatorname{Mod}_{g}\left(\right.$ resp. $\left.\operatorname{Mod}_{g, 1}\right)$ is at least exponential and at most doubly exponential.

We conjecture that the upper bound in Theorem 1.1 is, like the lower bound, exponential. The upper bound is strongly related to the isoperimetric and isodiametric inequalities in $\operatorname{Sp}(2 g, \mathbb{Z})$. Thurston has conjectured that for $n \geq 4$ the group $\operatorname{SL}(n, \mathbb{Z})$ satisfies a quadratic isoperimetric inequality. The analogous conjecture for $\operatorname{Sp}(2 g, \mathbb{Z})$ (together with a theorem of Papasoglu) would imply our conjectured upper bound. See $\S 3$ below, especially Remark 3.1, for a discussion.

For mapping class groups of surfaces with boundary components or punctures, one can construct finitely generated, normal subgroups by "filling in the punctures and boundary components"; these are the so-called surface braid groups (see [Bir]). For example, the point pushing subgroup $\pi_{1}\left(\Sigma_{g}\right) \triangleleft \operatorname{Mod}_{g}^{1}$ is the kernel of the surjection $\operatorname{Mod}_{g}^{1} \rightarrow \operatorname{Mod}_{g}$ induced by "filling in the puncture".

Theorem 1.2 (Distortion of surface braid groups). Let $K$ be the kernel of the surjection

$$
\operatorname{Mod}_{g, b+n}^{p+m} \rightarrow \operatorname{Mod}_{g, b}^{p},
$$

where at least one of $n$ and $m$ is strictly greater than 0 . Then $K$ is exactly exponentially distorted in $\operatorname{Mod}_{g, b+n}^{p+m}$ for $g \geq 2$.

We prove Theorem 1.2 in $\S 4$. 
In $\$ 5.2$ we construct "relative Johnson homomorphisms", relative to arbitrary subgroups of $\mathrm{H}_{1}\left(\Sigma_{g} ; \mathbb{Z}\right)$. Applying these homomorphisms together with the general method for obtaining lower bounds on distortion given in $\$ 2.2$, we obtain Theorems 1.3 and 1.5 below as well as a general result, Theorem 5.12, given in $§ 5.2$.

Theorem 1.3 (Distortion of the Lagrangian subgroup). For $b \in\{0,1\}$, let $L<$ $\operatorname{Mod}_{g, b}$ be the Lagrangian subgroup, that is, the group of mapping classes which act trivially on a fixed maximal isotropic subgroup of $\mathrm{H}_{1}\left(\Sigma_{g, b} ; \mathbb{Z}\right)$. Then $L$ is at least exponentially distorted in $\operatorname{Mod}_{g, b}$ for $g \geq 4$.

Remark 1.4. The Lagrangian subgroup was first defined by Garoufalidis and Levine [GL] and plays an important role in the theory of finite-type invariants of 3-manifolds.

Masur-Minsky [MM] (see [Ham1] for an alternate proof) proved that for $h<g$ the natural inclusion $\operatorname{Mod}_{h, 1} \hookrightarrow \operatorname{Mod}_{g}$ given by subsurface inclusion $\Sigma_{h, 1} \hookrightarrow \Sigma_{g}$ is linearly distorted ("undistorted" in their terminology). We also remark that, as a consequence of [LMR], if $1<h<g$ then $\operatorname{Sp}(2 h, \mathbb{Z})$ is linearly distorted in $\operatorname{Sp}(2 g, \mathbb{Z})$. One might therefore expect that the subgroup of $\operatorname{Mod}_{g}$ consisting of mapping classes "homologically supported" on $\Sigma_{h, 1}$ have linear distortion. In contrast we have the following.

Theorem 1.5. Suppose $g-h \geq 2$. Let $\Sigma_{h, 1}$ be an embedded subsurface of $\Sigma_{g}$, and let $K$ be the pull-back to $\operatorname{Mod}_{g}$ of the corresponding copy of $\mathrm{Sp}(2 h, \mathbb{Z})$ in $\mathrm{Sp}(2 g, \mathbb{Z})$. Then $K$ is at least exponentially distorted in $\operatorname{Mod}_{g}$.

We would like to know what happens when $g=h+1$, as our methods do not seem to work in this case.

A first idea. The first key idea in this paper can be illustrated by the following proof sketch of the lower bound in Theorem 1.1 in the case of $\mathcal{I}_{g}$ with $g \geq 3$. We begin with the standard exact sequence

$$
1 \longrightarrow \mathcal{I}_{g} \longrightarrow \operatorname{Mod}_{g} \longrightarrow \operatorname{Sp}(2 g, \mathbb{Z}) \longrightarrow 1
$$

coming from the action of $\operatorname{Mod}_{g}$ on $H:=\mathrm{H}_{1}\left(\Sigma_{g} ; \mathbb{Z}\right) \cong \mathbb{Z}^{2 g}$. Let $U=\bigwedge^{3} H / H$. Johnson proved in [Jo1] that there is a surjective homomorphism

$$
\tau: I_{g} \rightarrow U
$$

which is equivariant with respect to the natural actions of $\operatorname{Mod}_{g}$. Here the $\operatorname{Mod}_{g}$ action on $U$ factors through the standard $\operatorname{Sp}(2 g, \mathbb{Z})$ action, and the $\operatorname{Mod}_{g}$ action on $\mathcal{I}_{g}$ is the one induced by conjugation. This equivariance is just the formula

$$
\tau\left(f h f^{-1}\right)=f_{*} \tau(h) \quad \text { for all } f \in \operatorname{Mod}_{g}, h \in \mathcal{I}_{g},
$$

where $f_{*}$ denotes the induced action of $f \in \operatorname{Mod}_{g}$ on $\mathrm{H}_{1}\left(\Sigma_{g} ; \mathbb{Z}\right)$. 
We can use $\tau$ to give lower bounds for word length in $\mathcal{I}_{g}$ as follows. Fix a finite generating set $S$ for $\mathcal{I}_{g}$, and let $\|h\|_{\mathcal{I}_{g}}$ denote word length of $h$ with respect to $S$, that is, the minimal number of elements of $S^{ \pm 1}$ whose product equals $h$. Also, fix a norm $\|\cdot\|_{U}$ on $U$. Since $S$ is finite, there exists some $C>0$ such that $\|\tau(s)\|_{U} \leq C$ for all $s \in S$. Since $\tau$ is a homomorphism, it is then clear that for every $h \in \mathcal{I}_{g}$ we have

$$
\|h\|_{\mathcal{I}_{g}} \geq \frac{1}{C}\|\tau(h)\|_{U} .
$$

Now it is not hard to see that we can choose a mapping class $f \in \operatorname{Mod}_{g}$ with the property that the linear transformation $f_{*} \in \mathrm{GL}(U)$ is partially hyperbolic, that is, there is some eigenvalue $\lambda$ of $f_{*}$ with $|\lambda|>1$. Using the partial hyperbolicity of $f$, we will find an $h \in \mathcal{I}_{g}$ such that $\left\|f_{*}^{n}(\tau(h))\right\|_{U}$ grows exponentially in $n$. Then $\left\|f^{n} h f^{-n}\right\|_{\operatorname{Mod}_{g}}$ grows at most linearly with respect to $n$, but by (1) and (2) we have

$$
\begin{aligned}
\left\|f^{n} h f^{-n}\right\|_{\mathcal{I}_{g}} & \geq \frac{1}{C}\left\|\tau\left(f^{n} h f^{-n}\right)\right\|_{U} \\
& =\frac{1}{C}\left\|f_{*}^{n}(\tau(h))\right\|_{U},
\end{aligned}
$$

which grows exponentially. See $\S 3$ for details.

Some questions. This paper is a first attempt at a systematic study of distortion in mapping class groups. While the methods here apply to many examples, there are also many examples to which they do not apply. Of the many natural questions one might ask, we would like to point out a particular one to which our methods do not seem to apply. Recall that the handlebody group is the subgroup of $\operatorname{Mod}_{g}$ consisting of elements which extend to a fixed handlebody. Suzuki [Suz] proved that this group is finitely generated.

Question 1.6. What is the distortion of the handlebody subgroup in the mapping class group?

A natural question analogous to the direction of this paper is to study and compute the distortions of orbits of subgroups $K<\operatorname{Mod}_{g}$ in the Teichmüller space $\mathcal{T}_{g}$ for $\Sigma_{g}$, say endowed with the Teichmüller metric; see $[\mathrm{FM}],[\mathrm{KL}]$ for related discussions. Theorem 2.1 of [FLM] (and the discussion following it) give that any $\mathrm{Mod}_{g}$-orbit in $\mathcal{T}_{g}$ is exponentially distorted. Thus the problem of computing the distortion of $K$ in $\operatorname{Mod}_{g}$ and of a $K$-orbit in $\mathcal{T}_{g}$ are a priori different. We believe both questions are worth pursuing.

Acknowledgments. We are grateful to Danny Calegari for his help in refining the picture for Proposition 2.12, to Jordan Ellenberg for a helpful remark on the proof of Proposition 2.6, and to Hanna Bennett for pointing out an error in a previous version of this paper. We also thank the referee for several useful comments and corrections. 


\section{Methods for bounding distortion in groups}

In this section, we give two general methods for bounding the distortion of one group inside another, one yielding lower bounds and the other upper bounds. We will apply these methods throughout this paper to subgroups of mapping class groups.

2.1. Behavior of distortion under inclusions. Before we begin, we will need to know the following basic property of distortion.

Lemma 2.1 (Behavior of distortion under inclusions). Let $K<\Gamma$ be finitely generated groups. If $\Gamma<\Gamma^{\prime}$ with $\Gamma^{\prime}$ finitely generated, then the distortion of $K$ in $\Gamma^{\prime}$ is at least the distortion of $K$ in $\Gamma$.

Proof. Fix a finite generating set $S_{\Gamma}$ for $\Gamma$ and let $\|\cdot\|_{\Gamma}$ be the associated word metric. We can choose a finite generating set $S_{\Gamma^{\prime}}$ for $\Gamma^{\prime}$ with $S_{\Gamma} \subset S_{\Gamma^{\prime}}$. Letting $\|\cdot\|_{\Gamma^{\prime}}$ be the associated word metric, we have $\|k\|_{\Gamma} \geq\|k\|_{\Gamma^{\prime}}$ for all $k \in K$, and the lemma follows.

2.2. Lower bounds via irreducible representations. If $K<G$, then to give a lower bound for the distortion of $K$ in $G$, one must be able to give lower bounds on the word length in $K$. This is in general a very difficult problem, but for free abelian groups $V$ such bounds can be easily obtained. Moreover, the resulting linear algebra is a rich source of examples of exponential growth. We say that an element of the automorphism group $\mathrm{GL}(V):=\operatorname{Aut}(V)$ is partially hyperbolic if the corresponding linear transformation of $V \otimes \mathbb{C}$ has some eigenvalue $\lambda$ with $|\lambda|>1$. We then have the following easy example of exponential distortion.

Example 2.2. Let $\Gamma$ be the semidirect product of $V=\mathbb{Z}^{n}$ with any subgroup of $\operatorname{SL}(n, \mathbb{Z})$ which contains a partially hyperbolic matrix $A$. Then $V$ has exponential distortion in $\Gamma$. The upper bound is easy. The exponential lower bound follows, as explained in the "First idea" on page 540 and given in detail in Proposition 2.3 below, from the exponential growth of the image of vectors in $V \otimes \mathbb{R}$ under iteration of $A$.

To generalize this example, we will map group/subgroup pairs to mapping tori of abelian groups like those in Example 2.2, using the abelian group as a sort of "detector" of exponential distortion.

Proposition 2.3 (Criterion for exponential distortion). Let $K$ be a finitely generated normal subgroup of a finitely generated group $\Gamma$. Suppose that $V$ is a free abelian group equipped with a $\Gamma$-action $\rho: \Gamma \rightarrow \mathrm{GL}(V)$ and that $\psi: K \rightarrow V$ is a surjective homomorphism which is $\Gamma$-equivariant, where $\Gamma$ acts on $K$ by conjugation. If $\rho(\Gamma)$ contains a partially hyperbolic matrix, then the distortion of $K$ in $\Gamma$ is at least exponential. 
Remark 2.4. When applying Proposition 2.3, we will find it useful to think of the exact sequence

$$
1 \longrightarrow K \longrightarrow \Gamma \longrightarrow \Gamma / K \longrightarrow 1
$$

and to note that since $V$ is abelian and $\psi: K \rightarrow V$ is surjective we have

$$
\rho(k) \cdot \psi(y)=\psi\left(k y k^{-1}\right)=\psi(k)+\psi(y)-\psi(k)=\psi(y) \quad \text { for all } k, y \in K \text {. }
$$

Thus the action of $K$ on $V$ is trivial. Hence $\rho$ factors through a representation $\Gamma / K \rightarrow \mathrm{GL}(V)$.

Remark 2.5. By using Lemma 2.1, Proposition 2.3 can be applied beyond the context of normal subgroups. See the proofs of Theorem 1.3 and Theorem 1.5 for examples.

Proof of Proposition 2.3. The hypothesis that $\psi$ is $\Gamma$-equivariant is precisely that

$$
\psi\left(x y x^{-1}\right)=\rho(x) \cdot \psi(y) \quad \text { for all } y \in K \text { and } x \in \Gamma .
$$

By assumption, there exists some $x \in \Gamma$ such that

$$
\rho(x) \otimes 1: V \otimes \mathbb{C} \rightarrow V \otimes \mathbb{C}
$$

has an eigenvalue $\lambda_{1}$ with $\left|\lambda_{1}\right|>1$. Let $M=\rho(x) \otimes 1$ and let the distinct eigenvalues of $M$ be $\lambda_{1}, \ldots, \lambda_{k} \in \mathbb{C}$. For $1 \leq i \leq k$, let $E_{k}$ be the generalized eigenspace for $\lambda_{i}$, i.e. the kernel of the map $\left(M-\lambda_{i} \mathbb{I}\right)^{\operatorname{dim}(V)}$, where $\mathbb{I}$ is the identity. We have a direct sum decomposition $V \otimes \mathbb{C}=E_{1} \oplus \cdots \oplus E_{k}$ which is invariant under $M$. Endow $V \otimes \mathbb{C}$ with an inner product such that the $E_{i}$ are orthogonal to each other and let $\|\cdot\|$ be the associated norm. It is well-known (see, e.g., $[\mathrm{KH}], \S 2.2$ ) that there exists some $C>0$ such that if $v \in E_{1}$, then $\left\|M^{n} \cdot v\right\| \geq C\left|\lambda_{1}\right|^{n}\|v\|$ for all $n \geq 1$.

Let $S$ be a finite generating set for $K$ and let $S^{\prime} \supseteq S$ be a finite generating set for $\Gamma$. Since $S$ is finite, there exists some $D \geq 0$ such that

$$
\|\psi(s) \otimes 1\| \leq D \quad \text { for each } s \in S .
$$

Since $\psi$ is surjective, its image in $V \otimes \mathbb{C}$ must span $V \otimes \mathbb{C}$. In particular, its image cannot lie in $E_{2} \oplus \cdots \oplus E_{k}$, so we can find some $y \in K$ such that $\psi(y) \otimes 1 \notin$ $E_{2} \oplus \cdots \oplus E_{k}$. Let $y_{1}$ be the orthogonal projection of $\psi(y) \otimes 1$ to $E_{1}$. Using the fact that the $E_{i}$ are orthogonal and invariant under $M$, we deduce that for all $n \geq 1$ we have

$$
\begin{aligned}
\left\|\psi\left(x^{n} y x^{-n}\right) \otimes 1\right\| & =\left\|M^{n} \cdot(\psi(y) \otimes 1)\right\| \\
& \geq\left\|M^{n} \cdot y_{1}\right\| \\
& \geq C\left|\lambda_{1}\right|^{n}\left\|y_{1}\right\| .
\end{aligned}
$$


Now (4) together with (5) implies that the word length $\left\|x^{n} y x^{-n}\right\|_{K}$ with respect to the generating set $S$ is at least $\frac{1}{D} \cdot C\left|\lambda_{1}\right|^{n}\left\|y_{1}\right\|$, which grows exponentially in $n$ since $\left|\lambda_{1}\right|>1$ and $y_{1} \neq 0$. On the other hand, since $x$ and $y$ have fixed word length with respect to the generating set $S^{\prime}$ for $\Gamma$, we see that the word length in $\Gamma$ of $x^{n} y x^{-n}$ grows at most linearly in $n$. This completes the proof of the proposition.

The following (purely linear-algebra) proposition gives a useful way in practice to prove that an action contains some partially hyperbolic matrix.

Proposition 2.6 (Representation-theoretic criterion for hyperbolicity). Let $\Gamma$ be $a$ group and let $V$ be a free abelian group equipped with a $\Gamma$-action. Suppose that $V$ contains a nontrivial $\Gamma$-submodule $W$ satisfying the following two properties:

(1) $W$ is irreducible in the sense that if $W^{\prime}<W$ is a nontrivial $\Gamma$-submodule, then $W^{\prime} \otimes \mathbb{R}=W \otimes \mathbb{R}$.

(2) The image of the map $v: \Gamma \rightarrow \mathrm{GL}(W)$ is infinite.

Then $v(\Gamma)$ contains a partially hyperbolic matrix.

Remark 2.7. In all the examples we consider in this paper, one could prove that the relevant representations contain partially hyperbolic matrices by hand; however, we believe that Proposition 2.6 gives a conceptual reason for the ubiquity of representations with this property.

Proof of Proposition 2.6. Choosing a basis, we can identify $\operatorname{GL}(W)$ with $\operatorname{GL}(m, \mathbb{Z})$ for some $m \geq 1$. If a matrix $A \in \mathrm{GL}(W)$ is not partially hyperbolic, then every eigenvalue $\lambda$ of $A$ satisfies $|\lambda|=1$. Since $A$ has integer entries, each of its eigenvalues is an algebraic integer. An old theorem of Kronecker (see, e.g., [Gr]) states that if $\lambda$ is any algebraic integer with $|\lambda|=1$ and with $\left|\lambda^{\prime}\right|=1$ for every Galois conjugate $\lambda^{\prime}$ of $\lambda$, then $\lambda$ is a root of unity.

Let $\operatorname{GL}(n, \mathbb{Z})[L]$ denote the level $L$ congruence subgroup of $\operatorname{GL}(n, \mathbb{Z})$, which consists of those $A \in \operatorname{GL}(n, \mathbb{Z})$ for which, when the entries of $A$ are taken $\bmod L$, the result is the identity matrix. Theorem 5.61 of [Mo] states that there exists some $L>1$ such that no eigenvalue of any element of $\operatorname{GL}(n, \mathbb{Z})[L]$ is a nontrivial root of unity. Pulling back $\operatorname{GL}(n, \mathbb{Z})[L]$ via $v$ then gives us a finite index subgroup $\Gamma^{\prime}$ of $\Gamma$ with the property that no eigenvalue of any element of $v\left(\Gamma^{\prime}\right)$ is a nontrivial root of unity. Since we are assuming (assumption (2)) that $v(\Gamma)$ is infinite, we know $v\left(\Gamma^{\prime}\right)$ is nontrivial (indeed infinite).

Suppose $v\left(\Gamma^{\prime}\right)$ contains no partially hyperbolic matrix. Then by the above two paragraphs, every $A \in v\left(\Gamma^{\prime}\right)$ must be unipotent, that is, $A$ has 1 as its only eigenvalue. But any subgroup of $\operatorname{GL}(n, \mathbb{R})$ consisting of unipotent matrices must be nilpotent, and indeed conjugate into the upper triangular group with 1's on the diagonal (see, 
e.g., Corollary 17.5 of $[\mathrm{Hu}])$. Setting

$$
U=\left\{w \in W \mid \Gamma^{\prime} \cdot w=w\right\},
$$

this implies that $U \neq 0$. Since $v(\Gamma)$ is infinite (assumption (2)), we have $U \neq W$, for otherwise $v\left(\Gamma^{\prime}\right)$ would be trivial and so $v(\Gamma)$ would be finite. Also, since $\Gamma^{\prime}$ is a normal subgroup of $\Gamma$, we have that $\Gamma \cdot U \subset U$; indeed, for $h \in \Gamma$ and $u \in U$, we have for all $g \in \Gamma^{\prime}$ that

$$
g \cdot(h \cdot u)=h \cdot\left(\left(h^{-1} g h\right) \cdot u\right)=h \cdot u,
$$

so $h \cdot u \in U$. We conclude that $v(\Gamma)$ is reducible, contradicting assumption (1). Thus $v\left(\Gamma^{\prime}\right) \subset v(\Gamma)$ must contain some partially hyperbolic matrix, and we are done.

To apply Proposition 2.6, we will need a method for establishing the irreducibility of the action of the discrete group $\Gamma$. In the cases which arise in this paper, this action will factor through various arithmetic subgroups (such as $\operatorname{Sp}(2 g, \mathbb{Z})$ ) of semisimple Lie groups, and hence the following theorem of Borel can be applied.

Theorem 2.8 ([Bor1], Proposition 3.2). Let $G$ be a connected, semisimple, real algebraic group which is defined over $\mathbb{Q}$ and which has no compact factors (for example, $G=\operatorname{Sp}(2 g, \mathbb{R})$ for $g \geq 1$ or $G=\operatorname{SL}(n, \mathbb{R})$ for $n \geq 2)$. Let $V$ be a finite dimensional vector space over $\mathbb{R}$ which is an irreducible $G$-module. Then $V$ is also an irreducible $G_{\mathbb{Z}}$-module, where $G_{\mathbb{Z}}$ is the group of integer points of $G$.

Remark 2.9. The conditions in [Bor1], Proposition 3.2, do not mention integer points, but instead refer to subgroups satisfying a certain "property (S)". However, it is easy to see that lattices satisfy this property, and a well-known theorem of BorelHarish-Chandra says that arithmetic subgroups are lattices. We also remark that Proposition 3.2 in [Bor1] is one of the steps in the proof of an early version of the Borel Density Theorem, but later proofs do not make use of this result, and Theorem 2.8 can be easily deduced from the Borel Density Theorem.

2.3. Isoperimetric inequalities and upper bounds on distortion. The goal of this subsection is to prove that an upper bound for the distortion of a normal subgroup $K \triangleleft \Gamma$ can be obtained from isoperimetric and isodiametric functions on the quotient group $\Gamma / K$. We first recall some definitions. While the notion of isoperimetric function and isodiametric function for a group are usually defined as independent quantities (see, e.g., [Ger]), we will be interested in their simultaneous realization, as discussed in [GR] and in [Ril], Definition 2.2.2. We refer the reader to these references for background on this topic. 
Definition 2.10 (Simultaneous (isoperimetric, isodiametric) pairs). Let $\Gamma \cong\langle S \mid R\rangle$ be a finitely-presented group. Denote the free group on $S$ by $F(S)$. Also, denote the word length of an element $w$ in $F(S)$ by $\ell(w)$. We say that a pair of functions $\left(\mu_{P}, \mu_{D}\right)$ is a simultaneous (isoperimetric, isodiametric) pair for $\Gamma$ if for all $w \in$ $F(S)$ representing the identity in $\Gamma$, we can write $w$ as a word

$$
w=\prod_{i=1}^{N} x_{i} r_{i} x_{i}^{-1}, \quad x_{i} \in F(S), r_{i} \in R
$$

such that

$$
N \leq \mu_{P}(\ell(w)) \text { and } \ell\left(x_{i}\right) \leq \mu_{D}(\ell(w)) \quad \text { for each } 1 \leq i \leq N .
$$

Remark 2.11. Though the precise functions in an (isoperimetric, isodiametric) pair for a group depend on the choice of finite generating set, changing the generators preserves the equivalence classes of these functions up to linear substitution. Thus as with distortion we will consider (isoperimetric, isodiametric) pairs only up to their well-defined equivalence classes.

The main result of this subsection is the following, which is a small generalization of a theorem of Arzhantseva and Osin ([AO], Lemma 3.6).

Proposition 2.12 (Upper bound on distortion). Let $K \triangleleft \Gamma$ be a finitely generated, normal subgroup of a finitely generated group $\Gamma$. Suppose that $\Gamma / K$ is finitely presented and that $\left(\mu_{P}, \mu_{D}\right)$ is a simultaneous (isoperimetric, isodiametric) pair for $\Gamma / K$. Then there exists some $C>0$ such that the distortion of $K$ in $\Gamma$ is at most $\mu_{P} C^{\mu_{D}}$.

Proof. Let $S_{\Gamma}$ and $S_{K}$ be finite generating sets for $\Gamma$ and $K$ respectively, and let $F\left(S_{\Gamma}\right)$ be the free group on $S_{\Gamma}$. Let $\|\cdot\|_{\Gamma}: \Gamma \rightarrow \mathbb{N}$ be the $S_{\Gamma}$ word metric and $\|\cdot\|_{K}: K \rightarrow \mathbb{N}$ be the $S_{K}$ word metric. Let $\ell: F\left(S_{\Gamma}\right) \rightarrow \mathbb{N}$ give the word length in $F\left(S_{\Gamma}\right)$ and $\pi: F\left(S_{\Gamma}\right) \rightarrow \Gamma$ be the natural projection. Finally, choose $R \subset F\left(S_{\Gamma}\right)$ such that $\Gamma / K \cong\left\langle S_{\Gamma} \mid R\right\rangle$ is a finite presentation.

We begin by claiming that there is some $C>0$ such that if $r \in R$ and $x \in F\left(S_{\Gamma}\right)$, then

$$
\left\|\pi\left(x r x^{-1}\right)\right\|_{K}<C^{\ell(x)} .
$$

Indeed, an easy induction shows that if

$$
C_{1}=\max \left\{\|\pi(r)\|_{K} \mid r \in R\right\},
$$

and

$$
C_{2}=\max \left\{\left\|\pi\left(s_{\Gamma} s_{K} s_{\Gamma}^{-1}\right)\right\|_{K} \mid s_{\Gamma} \in S_{\Gamma} \text { and } s_{K} \in S_{K}\right\},
$$


then $C=C_{1} C_{2}$ satisfies the claim.

Now given $k \in K$ choose an efficient word $w \in F\left(S_{\Gamma}\right)$ such that $\pi(w)=k$ and $\ell(w)=\|k\|_{\Gamma}$. By Definition 2.10, we can write

$$
w=\prod_{i=1}^{N} x_{i} r_{i} x_{i}^{-1}, \quad x_{i} \in F\left(S_{\Gamma}\right), r_{i} \in R
$$

with

$$
N \leq \mu_{P}(\ell(w)) \text { and } \ell\left(x_{i}\right) \leq \mu_{D}(\ell(w)) \text { for each } 1 \leq i \leq N .
$$

We then calculate:

$$
\begin{aligned}
\|k\|_{K} & =\|\pi(w)\|_{K} \leq \sum_{i=1}^{N}\left\|\pi\left(w_{i} r_{i} w_{i}^{-1}\right)\right\|_{K} \leq \sum_{i=1}^{N} C^{\mu_{D}(\ell(w))} \\
& \leq \mu_{P}(\ell(w)) C^{\mu_{D}(\ell(w))} \\
& =\mu_{P}\left(\|k\|_{\Gamma}\right) C^{\mu_{D}\left(\|k\|_{\Gamma}\right)},
\end{aligned}
$$

as desired.

\section{Distortion of the Torelli group}

In this section, we apply Propositions 2.3 and 2.12 to give lower and upper bounds on the distortion of $\mathcal{I}_{g, b}$ in $\operatorname{Mod}_{g, b}$ for $b \in\{0,1\}$ and $g \geq 3$.

Proof of Theorem 1.1. Consider the standard exact sequence

$$
1 \longrightarrow \mathcal{I}_{g, b} \longrightarrow \operatorname{Mod}_{g, b} \longrightarrow \operatorname{Sp}(2 g, \mathbb{Z}) \longrightarrow 1 .
$$

We begin with the lower bound. Set $H=\mathrm{H}_{1}\left(\Sigma_{g, b} ; \mathbb{Z}\right)$. In [Jo1], Johnson constructed the Johnson homomorphisms, which are for $g \geq 3$ surjective $\operatorname{Mod}_{g, b^{-}}$ equivariant homomorphisms

$$
\tau: \mathcal{I}_{g, 1} \rightarrow \bigwedge^{3} H
$$

and

$$
\tau: \mathcal{I}_{g} \rightarrow\left(\bigwedge^{3} H\right) / H
$$

Here $H$ is embedded in $\wedge^{3} H$ as $H \wedge \omega$, where $\omega=a_{1} \wedge b_{1}+\cdots+a_{g} \wedge b_{g}$ for any symplectic basis $\left\{a_{1}, b_{1}, \ldots, a_{g}, b_{g}\right\}$ of $H$. The action of $\operatorname{Mod}_{g, b}$ on $\left(\bigwedge^{3} H\right) / H$ or $\bigwedge^{3} H$ factors surjectively through the infinite group $\operatorname{Sp}(2 g, \mathbb{Z})$.

Now $H \otimes \mathbb{R}$ is an irreducible representation of $\operatorname{Sp}(2 g, \mathbb{R})$. By Theorem 2.8 it is also an irreducible $\operatorname{Sp}(2 g, \mathbb{Z})$-representation. We can apply Proposition 2.6 
to the inclusion $H \subset \wedge^{3} H$ to show that some element of $\operatorname{Mod}_{g, 1}$ acts partially hyperbolically on $\bigwedge^{3} H$. Similarly, $\left(\left(\bigwedge^{3} H\right) / H\right) \otimes \mathbb{R}$ is an irreducible representation of $\operatorname{Sp}(2 g, \mathbb{R})$. By Theorem 2.8 it is also an irreducible $\operatorname{Sp}(2 g, \mathbb{Z})$-representation. We can apply Proposition 2.6 with $W=V=\left(\bigwedge^{3} H\right) / H$ to show that some element of $\operatorname{Mod}_{g, 0}$ acts partially hyperbolically on $\left(\bigwedge^{3} H\right) / H$.

Now apply Proposition 2.3 to $\mathcal{I}_{g, b} \triangleleft \operatorname{Mod}_{g, b}$ with the homomorphism $\psi$ equal to the relevant Johnson homomorphism to conclude that $\mathcal{I}_{g, b}$ is at least exponentially distorted in $\operatorname{Mod}_{g, b}$ for $g \geq 3$ and $b \in\{0,1\}$, as desired.

We now establish the upper bound. As explained in $\$ 5$ of [Leu] (see in particular Corollary 5.4), the nonpositively curved symmetric space $X$ for $\operatorname{Sp}(2 g, \mathbb{R})$ admits an $\operatorname{Sp}(2 g, \mathbb{Z})$-equivariant retraction $r: X \rightarrow \Omega$ onto a submanifold with boundary $\Omega$ on which $\mathrm{Sp}(2 g, \mathbb{Z})$ acts cocompactly and properly by isometries (in the path metric). Now $X$ is a nonpositively curved Riemannian manifold, and so it has a simultaneous (isoperimetric, isodiametric) function which is (quadratic, linear). The retraction $r$ distorts lengths and hence volumes by at most an exponential factor. It follows that $\Omega$, hence $\operatorname{Sp}(2 g, \mathbb{Z})$, has at worst a simultaneous (exponential, exponential) (isoperimetric, isodiametric) pair. While [Leu] explains this only for isoperimetric functions, the argument for the (isoperimetric, isodiametric) pair follows exactly his argument.

Proposition 2.12 applied to exact sequence (6) therefore implies that for $g \geq 3$ and $b \in\{0,1\}$, the group $\mathcal{I}_{g, b}$ is at most doubly exponentially distorted in $\operatorname{Mod}_{g, b}$, as desired.

Remark 3.1. The isoperimetric/isodiametric bounds for $\operatorname{Sp}(2 g, \mathbb{Z})$ used in the proof of Theorem 1.1 are probably not sharp. In fact, Thurston has conjectured that $\operatorname{SL}(n, \mathbb{Z})$ satisfies a quadratic isoperimetric inequality for $n \geq 4$, and one would expect the same to hold for $\operatorname{Sp}(2 g, \mathbb{Z})$ for $g \geq 3$. If $\operatorname{Sp}(2 g, \mathbb{Z})$ satisfied a quadratic isoperimetric inequality, then a theorem of Papasoglu [Pap] (see [GR], Theorem 2, for a generalization and alternate proof) would imply that it has a (quadratic, linear) (isoperimetric, isodiametric) pair. This would imply that our exponential lower bound on the distortion of $\mathcal{I}_{g}$ in $\operatorname{Mod}_{g}$ (and of $\mathcal{I}_{g, 1}$ in $\operatorname{Mod}_{g, 1}$ ) is sharp.

In fact one could get by with much less than Thurston's conjecture. Sharpness of the exponential lower bound on the distortion of the Torelli group would follow if $\operatorname{Sp}(2 g, \mathbb{Z})$ satisfied an (exponential, linear) (isoperimetric, isodiametric) pair.

\section{Distortion of surface braid groups}

Proof of Theorem 1.2. Consider the exact sequence

$$
1 \longrightarrow K \longrightarrow \operatorname{Mod}_{g, b+n}^{p+m} \longrightarrow \operatorname{Mod}_{g, b}^{p} \longrightarrow 1 .
$$


We first prove that $K$ is at least exponentially distorted in $\operatorname{Mod}_{g, b+n}^{p+m}$. Setting $H=$ $\mathrm{H}_{1}\left(\Sigma_{g} ; \mathbb{Z}\right)$, we will construct a $\operatorname{Mod}_{g, b+n}^{p+m}$-equivariant surjection $\psi: K \rightarrow H^{n+m}$. Here the $\operatorname{Mod}_{g, b+n}^{p+m}$-action on $H^{n+m}$ factors through the natural projection

$$
\operatorname{Mod}_{g, b+n}^{p+m} \rightarrow \operatorname{Mod}_{g} \rightarrow \operatorname{Sp}(2 g, \mathbb{Z}) .
$$

Since $H^{n+m}$ contains the nontrivial irreducible (by Theorem 2.8) $\mathrm{Sp}(2 g, \mathbb{Z})$-module $H$, we can apply Propositions 2.6 and 2.3 to $K \triangleleft \operatorname{Mod}_{g, b+n}^{p+m}$ with the homomorphism $\psi$ to conclude that $K$ is at least exponentially distorted in $\operatorname{Mod}_{g, b+n}^{p+m}$, as desired.

The construction of $\psi$ goes as follows. Let $K^{\prime}$ be the kernel of the surjection $\operatorname{Mod}_{g, b}^{p+m+n} \rightarrow \operatorname{Mod}_{g, b}^{p}$. The map $\operatorname{Mod}_{g, b+n}^{p+m} \rightarrow \operatorname{Mod}_{g, b}^{p+m+n}$ induces a surjection $K \rightarrow K^{\prime}$. A basic result of Birman [Bir] shows that $K^{\prime}$ is the fundamental group of the configuration space of $n+m$ points on $\Sigma_{g+b}^{p}$, that is, that $K^{\prime} \cong \pi_{1}\left(\left(\Sigma_{g, b}^{p}\right)^{n+m} \backslash \Delta\right)$ where

$$
\Delta=\left\{\left(x_{1}, \ldots, x_{n+m}\right) \in\left(\Sigma_{g, b}^{p}\right)^{n+m} \mid x_{i}=x_{j} \text { for some } i \neq j\right\} .
$$

The map $\psi$ is then the composition

$$
K \rightarrow K^{\prime}=\pi_{1}\left(\left(\Sigma_{g, b}^{p}\right)^{n+m} \backslash \Delta\right) \rightarrow \pi_{1}\left(\left(\Sigma_{g, b}^{p}\right)^{n+m}\right) \rightarrow \pi_{1}\left(\left(\Sigma_{g}\right)^{n+m}\right) \rightarrow H^{n+m} .
$$

We now prove that $K$ is at most exponentially distorted in $\operatorname{Mod}_{g, b+n}^{p+m}$. In [Mos], Mosher proved that $\operatorname{Mod}_{g, b}^{p}$ is automatic. This implies [ECHLPT] that $\operatorname{Mod}_{g, b}^{p}$ satisfies a simultaneous (quadratic, linear) (isoperimetric, isodiametric) pair. We can thus deduce the desired upper bound by applying Proposition 2.12 to the exact sequence (7).

\section{Relative Johnson homomorphisms and the distortion of homologically specified subgroups}

In this section, we generalize the Johnson homomorphism to other subgroups of $\operatorname{Mod}_{g, b}$ defined by a variety of homological conditions. We then apply these homomorphisms to give lower bounds on distortion.

5.1. Relative Johnson homomorphisms. Fix $g \geq 3$ and $b \in\{0,1\}$, and set $H=$ $\mathrm{H}_{1}\left(\Sigma_{g, b} ; \mathbb{Z}\right)$. We will consider the following subgroups of the mapping class group $\operatorname{Mod}_{g, b}$.

Definition 5.1. Let $W$ be a subgroup of $H$. Define

$$
\operatorname{Mod}_{g, b}^{W}:=\left\{f \in \operatorname{Mod}_{g, b} \mid f_{*}(W) \subset W\right\} .
$$


Observe that $\operatorname{Mod}_{g, b}^{W}$ acts on $H / W$. The kernel of this action will be denoted by $\mathcal{I}_{g, b}^{W}$.

Example 5.2. The classical Torelli group $\mathcal{I}_{g, b}$ corresponds to $\mathcal{I}_{g, b}^{W}$ with $W=0$.

Example 5.3. Fix $m \in \mathbb{N}$, and let $W$ be the kernel of the map $H \rightarrow \mathrm{H}_{1}\left(\Sigma_{g, b} ; \mathbb{Z} / m \mathbb{Z}\right)$. Then $\operatorname{Mod}_{g, b}^{W}=\operatorname{Mod}_{g, b}$ and $\mathcal{I}_{g, b}^{W}$ is the level $m$ subgroup of $\operatorname{Mod}_{g, b}$, that is, the kernel of natural map $\operatorname{Mod}_{g, b} \rightarrow \operatorname{Sp}(2 g, \mathbb{Z} / m \mathbb{Z})$.

Example 5.4. Let $\Sigma^{\prime} \subset \Sigma_{g, b}$ be a subsurface, and let $W$ be the image of $\mathrm{H}_{1}\left(\Sigma^{\prime} ; \mathbb{Z}\right)$ in $H$. Then $\mathcal{I}_{g, b}^{W}$ is generated by $\mathcal{I}_{g, b}$ together with the set mapping classes supported on $\Sigma^{\prime}$. One may think of this as the subgroup of mapping classes which are "homologically supported" on $\Sigma^{\prime}$.

Example 5.5. Let $W \subset \mathrm{H}_{1}\left(\Sigma_{g, b} ; \mathbb{Z}\right)$ be a Lagrangian, that is, a maximal isotropic subgroup. Then $\mathcal{I}_{g, b}^{W}$ is the subgroup of mapping classes which preserve $W$ and act trivially on $H / W$. It is easy to see that $\mathcal{I}_{g, b}^{W}$ must also act trivially on $W$. In other words, these are the Lagrangian subgroups of Theorem 1.3.

We now wish to generalize the Johnson homomorphisms to the groups $\mathcal{I}_{g, b}^{W}$. We begin by discussing the appropriate target for these Johnson homomorphisms. We will need the following well-known lemma.

Lemma 5.6 ([Bro], Theorem V.6.4). If $A$ is an abelian group, then there is an injection $i: \wedge^{3} A \rightarrow \mathrm{H}_{3}(A ; \mathbb{Z})$. If $A$ is torsion-free, then $i$ is an isomorphism.

Now, using Lemma 5.6, the classical Johnson homomorphism on a surface with boundary is of the form

$$
\tau: \mathcal{I}_{g, 1} \rightarrow \bigwedge^{3} H \cong \mathrm{H}_{3}(H ; \mathbb{Z})
$$

The relative Johnson homomorphism on a surface with boundary will be of the form

$$
\tau^{W}: \mathcal{I}_{g, 1}^{W} \rightarrow \mathrm{H}_{3}(H / W ; \mathbb{Z})
$$

On a closed surface, the classical Johnson homomorphism is of the form

$$
\tau: \mathcal{I}_{g} \rightarrow\left(\bigwedge^{3} H\right) / H \cong \mathrm{H}_{3}(H ; \mathbb{Z}) / H
$$

Here $H$ is embedded in $\wedge^{3} H$ as $H \wedge \omega$, where $\omega=a_{1} \wedge b_{1}+\cdots+a_{g} \wedge b_{g}$ for any symplectic basis $\left\{a_{1}, b_{1}, \ldots, a_{g}, b_{g}\right\}$ of $H$. By Lemma 5.6, there is a copy of $\bigwedge^{3}(H / W)$ in $\mathrm{H}_{3}(H / W ; \mathbb{Z})$. For any subgroup $W$ of $H$, the aforementioned 
embedding of $H$ into $\bigwedge^{3} H$ therefore projects to a homomorphism

$$
H \hookrightarrow \bigwedge^{3} H \rightarrow \bigwedge^{3}(H / W) \hookrightarrow \mathrm{H}_{3}(H / W ; \mathbb{Z})
$$

whose image we will denote by $H^{W}$. The relative Johnson homomorphism on a closed surface will be of the form

$$
\tau^{W}: \mathcal{I}_{g}^{W} \rightarrow \mathrm{H}_{3}(H / W ; \mathbb{Z}) / H^{W} .
$$

Remark 5.7. The map $H \rightarrow H^{W}$ need not be injective. Indeed, if $W$ is a maximal isotropic subspace of $H$, then the image of $\omega$ in $\bigwedge^{2}(H / W)$ is 0 . Hence in this case $H^{W}=0$.

For simplicity, we will define $H^{W}=0$ if $b=1$. We can now state our theorem.

Theorem 5.8 (Relative Johnson homomorphisms). For $g \geq 3$ and $b \in\{0,1\}$, there exist homomorphisms

$$
\tau^{W}: \mathcal{I}_{g, b}^{W} \rightarrow \mathrm{H}_{3}(H / W ; \mathbb{Z}) / H^{W},
$$

which we will call the relative Johnson homomorphisms, satisfying the following properties:

(1) $\tau^{0}$ is the classical Johnson homomorphism.

(2) If $W_{1} \subset W_{2}$, then $\left.\tau^{W_{2}}\right|_{\mathcal{I}_{g, b}^{W_{1}}}$ equals $\tau^{W_{1}}$ followed by the natural map

$$
\mathrm{H}_{3}\left(H / W_{1} ; \mathbb{Z}\right) / H^{W_{1}} \rightarrow \mathrm{H}_{3}\left(H / W_{2} ; \mathbb{Z}\right) / H^{W_{2}} .
$$

(3) For $f \in \operatorname{Mod}_{g, b}^{W}$ and $h \in \mathcal{I}_{g, b}$, we have $\tau\left(f h f^{-1}\right)=f_{*} \circ \tau(h)$, where $f_{*}$ is the induced map on $\mathrm{H}_{3}(H / W ; \mathbb{Z}) / H^{W}$.

Proof. We begin with the case $b=1$ (where $H^{W}=0$ ). As described for example in [Jo2], the classical Johnson homomorphism $\tau$ can be defined in various ways. We imitate the construction based on mapping tori. For a detailed discussion of this definition of the classical Johnson homomorphism and a proof of its equivalence to the more standard definition, see $\$ 3-4$ of [Hai]. For $h \in I_{g, 1}^{W}$, choose a homeomorphism $h^{\prime}$ of $\Sigma_{g, 1}$ representing $h$ and construct a homeomorphism $\bar{h}$ of $\Sigma_{g}$ by gluing a disc $D$ to the boundary component of $\Sigma_{g, 1}$ to get a copy of $\Sigma_{g}$ and defining $\bar{h}$ to equal 1 on $D$ and $h^{\prime}$ on $\Sigma_{g, 1}=\Sigma_{g} \backslash D$. Next, let $M_{\bar{h}}$ be the mapping torus of $\bar{h}$, that is, the quotient of $\Sigma_{g} \times[0,1]$ by the equivalence relation $(x, 1) \sim(\bar{h}(x), 0)$. Fixing a basepoint $v$ on $D \times 0 \subset \Sigma_{g} \times 0$, we get a canonical loop $\ell \in \pi_{1}\left(M_{\bar{h}}, v\right)$, via $\ell(t)=v \times t$. Observe that

$$
\mathrm{H}_{1}\left(M_{\bar{h}} ; \mathbb{Z}\right) /\langle\ell\rangle \cong H /\left\langle\left\{\alpha-h_{*} \alpha \mid \alpha \in H\right\}\right\rangle .
$$


Since $h \in \mathcal{I}_{g, 1}^{W}$, this has a natural projection to $H / W$. We conclude that there is a natural map

$$
\pi_{1}\left(M_{\bar{h}}, v\right) \rightarrow H / W
$$

and since $M_{\bar{h}}$ is an Eilenberg-MacLane space, we have an induced map

$$
\phi: M_{\bar{h}} \rightarrow K(H / W, 1)
$$

We define

$$
\tau^{W}(h)=\phi_{*}\left(\left[M_{\bar{h}}\right]\right) \in \mathrm{H}_{3}(H / W ; \mathbb{Z}) .
$$

It is easy to see that this definition is independent of the choices involved in its construction, and $\tau^{W}$ clearly satisfies Condition 2 of the theorem. Moreover, if $W=0$, then $\tau^{W}$ reduces to Johnson's definition of the classical Johnson homomorphism via mapping tori, so Condition 1 follows. The proof (see [Jo2]) that the classical Johnson homomorphism is a homomorphism which satisfies Condition 3 generalizes verbatim to our situation, so we are done.

We now deal with the case $b=0$. We have an exact sequence

$$
1 \longrightarrow \pi_{1}\left(T^{1} \Sigma_{g}\right) \longrightarrow \mathcal{I}_{g, 1}^{W} \longrightarrow \mathcal{I}_{g}^{W} \longrightarrow 1
$$

where $T^{1} \Sigma_{g}$ is the unit tangent bundle of $\Sigma_{g}$; see [Jo3]. The classical Johnson homomorphism restricted to $\pi_{1}\left(T^{1} \Sigma_{g}\right)$ lands in $H \subset \wedge^{3} H$. It follows that $\tau^{W}$ restricted to $\pi_{1}\left(T^{1} \Sigma_{g}\right)$ lands in $H^{W} \subset \wedge^{3} H / W$, and thus we have an induced map

$$
\mathcal{I}_{g}^{W} \rightarrow\left(\mathrm{H}_{3}(H / W ; \mathbb{Z})\right) / H^{W} .
$$

These maps clearly satisfy the conditions of the theorem.

Remark 5.9. In the special case of the Lagrangian subgroup, Levine [Lev] has given a different construction of $\tau^{W}$. His construction imitates Johnson's original definition of $\tau$ [Jo2], First Definition, while our construction is inspired by Johnson's definition in terms of mapping tori [Jo2], Second Definition.

Remark 5.10. Another approach sufficient for the proofs of Theorems 1.3 and 1.5 would be to use Morita's [Mor1] extension of the classical Johnson homomorphism to a crossed homomorphism $\operatorname{Mod}_{g, 1} \rightarrow \frac{1}{2} \bigwedge^{3} H$.

5.2. Applications to subgroup distortion. We now apply the relative Johnson homomorphisms $\tau^{W}$ together with Propositions 2.6 and 2.3 to give lower bounds on the distortions of some of the groups $\mathcal{I}_{g, b}^{W}$ inside $\operatorname{Mod}_{g, b}$. For these distortions to make sense, however, we must first prove the following.

Proposition 5.11. For $g \geq 3$ and $b \in\{0,1\}$, let $W$ be any subgroup of $\mathrm{H}_{1}\left(\Sigma_{g, b} ; \mathbb{Z}\right)$. Then the groups $\mathcal{I}_{g, b}^{W}$ and $\operatorname{Mod}_{g, b}^{W}$ are finitely generated. 
Proof. Let $\Gamma$ be either $\mathcal{I}_{g, b}^{W}$ or $\operatorname{Mod}_{g, n}^{W}$. We have an exact sequence

$$
1 \longrightarrow \mathcal{I}_{g, b} \longrightarrow \Gamma \longrightarrow B \longrightarrow 1,
$$

where $B$ is the image of $\Gamma<\operatorname{Mod}_{g, b}$ in $\operatorname{Sp}(2 g, \mathbb{Z})$. As has already been mentioned, Johnson [Jo3] showed that $\mathcal{I}_{g, b}$ is finitely generated. It is enough, therefore, to show that $B$ is finitely generated. To see this, first note that $B$ is the set of $\mathbb{Z}$-points of the $\mathbb{Q}$-algebraic group consisting of matrices in $\operatorname{SL}(2 g, \mathbb{Q})$ which preserve the (integral) subgroup $W<\mathbb{Q}^{2 g}$ and the symplectic form and (for $\Gamma=\mathcal{I}_{g, b}^{W}$ ) which act trivially on $H / W$. Hence $B$ is arithmetic, and as such it is finitely generated (see, e.g. [Mo], Theorem 5.57).

We now prove Theorems 1.3 and 1.5 from the introduction.

Proof of Theorem 1.3. Let $H=\mathrm{H}_{1}\left(\Sigma_{g, b} ; \mathbb{Z}\right)$ with symplectic basis $\left\{a_{1}, \ldots, a_{g}\right.$, $\left.b_{1}, \ldots, b_{g}\right\}$ and let $W=\left\langle a_{1}, \ldots a_{g}\right\rangle$ be the standard Lagrangian. Then the Lagrangian subgroup $L$ is $\mathcal{I}_{g, b}^{W}$. By Lemma 2.1 and Proposition 5.11, to prove that $\mathcal{I}_{g, b}^{W}$ is at least exponentially distorted in $\operatorname{Mod}_{g, b}$, it is enough to prove that $\mathcal{I}_{g, b}^{W}$ is at least exponentially distorted in $\operatorname{Mod}_{g, b}^{W}$. The proof is based on the exact sequence

$$
1 \longrightarrow \mathcal{I}_{g, b}^{W} \longrightarrow \operatorname{Mod}_{g, b}^{W} \longrightarrow A \longrightarrow 1,
$$

where $A<\mathrm{Sp}(2 g, \mathbb{Z})$ consists of symplectic matrices with a $g \times g$ block of zeros in the lower left-hand corner. Now, by Remark 5.7, we have $H^{W}=0$. By Lemma 5.6, the relative Johnson homomorphism $\tau^{W}$ given by Theorem 5.8 is of the form

$$
\tau^{W}: \mathcal{I}_{g, b}^{W} \rightarrow \mathrm{H}_{3}(H / W ; \mathbb{Z}) \cong \bigwedge^{3}(H / W) \cong \bigwedge^{3}\left\langle b_{1}, \ldots, b_{g}\right\rangle .
$$

Moreover, it is easy to see that $A$ acts on $\mathrm{H}_{3}(H / W ; \mathbb{Z})$ via the surjection $A \rightarrow$ $\operatorname{SL}(g, \mathbb{Z})$ given by projection to the lower right hand $g \times g$ block. For $g \geq 4$, Theorem 2.8 says that the $A$-module $\wedge^{3} \mathbb{Z}^{g}$ is nontrivial and irreducible, so we can apply Propositions 2.6 and 2.3 to $\mathcal{I}_{g, b}^{W} \triangleleft \operatorname{Mod}_{g, b}^{W}$, setting $\psi=\tau^{W}$, to conclude that $\mathcal{I}_{g, b}^{W}$ is at least exponentially distorted in $\operatorname{Mod}_{g, b}^{W}$, as desired.

Proof of Theorem 1.5. First note that $K=I_{g}^{W}$, where $W=\mathrm{H}_{1}\left(\Sigma_{h, 1} ; \mathbb{Z}\right)$. Again, by Lemma 2.1 and Proposition 5.11, to prove that $\mathcal{I}_{g}^{W}$ is at least exponentially distorted in $\operatorname{Mod}_{g}$, it is enough to prove that $\mathcal{I}_{g}^{W}$ is at least exponentially distorted in $\operatorname{Mod}_{g}^{W}$. To prove this we consider the exact sequence

$$
1 \longrightarrow I_{g}^{W} \longrightarrow \operatorname{Mod}_{g}^{W} \longrightarrow A \longrightarrow 1,
$$

where $A<\operatorname{Sp}(2 g, \mathbb{Z})$ is isomorphic to $\operatorname{Sp}(2(g-h), \mathbb{Z})$. Now, by Lemma 5.6, the homomorphism $\tau^{W}$ given by Theorem 5.8 is of the form

$$
\tau^{W}: I_{g}^{W} \rightarrow \mathrm{H}_{3}(H / W ; \mathbb{Z}) / H^{W} \cong\left(\bigwedge^{3} \mathrm{H}_{1}\left(\Sigma_{g-h, 1} ; \mathbb{Z}\right)\right) / \mathrm{H}_{1}\left(\Sigma_{g-h, 1} ; \mathbb{Z}\right) .
$$


Since $g-h \geq 2$, Theorem 2.8 says that the $\operatorname{Sp}(2(g-h), \mathbb{Z})$-module

$$
\left(\bigwedge^{3} \mathrm{H}_{1}\left(\Sigma_{g-h, 1} ; \mathbb{Z}\right)\right) / \mathrm{H}_{1}\left(\Sigma_{g-h, 1}, \mathbb{Z}\right)
$$

is nontrivial and irreducible. Applying Propositions 2.6 and 2.3 to $\mathcal{I}_{g}^{W} \triangleleft \operatorname{Mod}_{g}^{W}$ with $\psi=\tau^{W}$ then gives that $\mathcal{I}_{g}^{W}$ is at least exponentially distorted in $\operatorname{Mod}_{g}^{W}$, as desired.

The following theorem gives lower bounds for the distortion of $\mathcal{I}_{g, b}^{W}$ in $\operatorname{Mod}_{g, b}$ for arbitrary $W$. As the proof is similar to the proofs of Theorems 1.3 and 1.5 , we omit it.

Theorem 5.12. Let $g \geq 3, b \in\{0,1\}$, and $W \subset H$. Assume that some element of $\operatorname{Mod}_{g, b}^{W}$ acts partially hyperbolically on $\mathrm{H}_{3}(H / W ; \mathbb{Z}) / H^{W}$. Then $\mathcal{I}_{g, b}^{W}$ is at least exponentially distorted in $\operatorname{Mod}_{g, b}$.

\section{References}

[AO] G. N. Arzhantseva and D. V. Osin, Solvable groups with polynomial Dehn functions. Trans. Amer. Math. Soc. 354 (2002), no. 8, 3329-3348 Zbl 0998.20040 MR 1897402546

[Bir] J. S. Birman, Braids, links, and mapping class groups. Ann. of Math. Stud. 82, Princeton University Press, Princeton, N.J., 1974. Zbl 0305.57013 MR 0375281 539,549

[Bor1] A. Borel, Density properties for certain subgroups of semi-simple groups without compact components. Ann. of Math. (2) 72 (1960), 179-188. Zbl 0094.24901 MR 0123639545

[Bro] K. S. Brown, Cohomology of groups. Corrected reprint of the 1982 original, Grad. Texts in Math. 87, Springer-Verlag, New York 1994. Zbl 0584.20036 MR 1324339 550

[ECHLPT] D. B. A. Epstein, J. W. Cannon, D. F. Holt, S. V. Levy, M. S. Paterson, and W. P. Thurston, Word processing in groups. Jones and Bartlett, Boston, MA, 1992. Zbl 0764.20017 MR 1161694549

[Far1] B. Farb, The extrinsic geometry of subgroups and the generalized word problem. Proc. London Math. Soc. (3) 68 (1994), no. 3, 577-593. Zbl 0816.20032 MR 1262309538

[Far2] B. Farb, Some problems on mapping class groups and moduli space. In Problems on mapping class groups and related topics, Proc. Sympos. Pure Math. 74, Amer. Math. Soc., Providence, RI, 2006, 11-55. Zbl 05124673 MR 2264130539

[FLM] B. Farb, A. Lubotzky, and Y. Minsky, Rank-1 phenomena for mapping class groups. Duke Math. J. 106 (2001), no. 3, 581-597. Zbl 1025.20023 MR 1813237 539, 541

[FM] B. Farb and L. Mosher, Convex cocompact subgroups of mapping class groups. Geom. Topol. 6 (2002), 91-152. Zbl 1021.20034 MR 1914566 539, 541 
[Ger] S. M. Gersten, Isoperimetric and isodiametric functions of finite presentations. In Geometric group theory, Vol. 1 (Sussex, 1991), Cambridge University Press, Cambridge 1993, 79-96. Zbl 0829.20054 MR 1238517545

[GL] S. Garoufalidis and J. Levine, Finite type 3-manifold invariants, the mapping class group and blinks. J. Differential Geom. 47 (1997), no. 2, 257-320. Zbl 0917.57009 MR 1601612540

[GR] S. M. Gersten and T. R. Riley, Filling length in finitely presentable groups. Geom. Dedicata 92 (2002), 41-58. Zbl 1040.20029 MR 1934008 545, 548

[Gr] G. Greiter, A simple proof for a theorem of Kronecker. Amer. Math. Monthly $\mathbf{8 5}$ (1978), no. 9, 756-757. MR 0514044544

[Gro] M. Gromov, Asymptotic invariants of infinite groups. In Geometric group theory, Vol. 2 (Sussex, 1991), Cambridge University Press, Cambridge 1993, 1-295. Zbl 0841.20039 MR 1253544 538

[Hai] R. M. Hain, Torelli groups and geometry of moduli spaces of curves. In Current topics in complex algebraic geometry (Berkeley, CA, 1992/93), Cambridge University Press, Cambridge 1995, 97-143. Zbl 0868.14006 MR 1397061551

[Ham1] U. Hamenstädt, Geometry of the mapping class groups II: (Quasi)-geodesics. Preprint, 2006. arXiv:0912.0137v1 [math.GR] 539, 540

[Ham2] U. Hamenstädt, Geometric properties of the mapping class group. in Problems on mapping class groups and related topics, Proc. Sympos. Pure Math. 74, Amer. Math. Soc., Providence, RI, 2006, 215-232. Zbl 05124685 MR 2264542539

[Hu] J.E. Humphreys, Linear algebraic groups. Grad. Texts in Math. 21, Springer-Verlag, New York 1975. Zbl 0325.20039 MR 0396773545

[Jo1] D. Johnson, An abelian quotient of the mapping class group $\mathcal{I}_{g}$. Math. Ann. 249 (1980), no. 3, 225-242. Zbl 0409.57009 MR 0579103 540, 547

[Jo2] D. Johnson, A survey of the Torelli group. In Low-dimensional topology (San Francisco, Calif., 1981), Contemp. Math. 20, Amer. Math. Soc., Providence, RI, 1983, 165-179. Zbl 0553.57002 MR 0718141 551, 552

[Jo3] D. Johnson, The structure of the Torelli group. I. A finite set of generators for $\mathcal{I}$. Ann. of Math. (2) 118 (1983), no. 3, 423-442. Zbl 0549.57006 MR 0727699 539, 552,553

[KH] A. Katok and B. Hasselblatt, Introduction to the modern theory of dynamical systems. Cambridge University Press, Cambridge, 1995. Zbl 0878.58020 MR 1326374 543

[KL] R. Kent and C. Leininger, Subgroups of mapping class groups from the geometrical viewpoint. In In the tradition of Ahlfors-Bers, IV, Contemp. Math. 432, Amer. Math. Soc., Providence, RI, 2007, 119-142. Zbl 1140.30017 MR 2342811541

[Lev] J. Levine, Pure braids, a new subgroup of the mapping class group and finite-type invariants of 3-manifolds. In Tel Aviv topology conference: Rothenberg Festschrift (1998), Contemp. Math. 231, Amer. Math. Soc., Providence, RI, 1999, 137-157. Zbl 0933.57018 MR 1705580552

[Leu] E. Leuzinger, On polyhedral retracts and compactifications of locally symmetric spaces. Differential Geom. Appl. 20 (2004), no. 3, 293-318. Zbl 1052.22008 MR 2053916548 
[LMR] A. Lubotzky, S. Mozes, and M. S. Raghunathan, The word and Riemannian metrics on lattices of semisimple groups. Inst. Hautes Études Sci. Publ. Math. 91 (2000), 5-53 (2001). Zbl 0988.22007 MR 1828742540

[MM] H. A. Masur and Y. N. Minsky, Geometry of the complex of curves. II. Hierarchical structure. Geom. Funct. Anal. 10 (2000), no. 4, 902-974. Zbl 0972.32011 MR 1791145 539, 540

[Mo] D. Witte Morris, Introduction to arithmetic groups. Preprint. http://people.uleth.ca/ dave.morris/books/IntroArithGroups.html 544, 553

[Mor1] S. Morita, The extension of Johnson's homomorphism from the Torelli group to the mapping class group. Invent. Math. 111 (1993), no. 1, 197-224. Zbl 0787.57008 MR 1193604552

[Mos] L. Mosher, Mapping class groups are automatic. Ann. of Math. (2) 142 (1995), no. 2, 303-384. Zbl 0867.57004 MR 1343324549

[Pap] P. Papasoglu, On the asymptotic cone of groups satisfying a quadratic isoperimetric inequality. J. Differential Geom. 44 (1996), no. 4, 789-806. Zbl 0893.20029 MR 1438192548

[Ril] T. Riley, Filling functions. In Geometry of the word problem for finitely generated groups, Adv. Courses Math. CRM Barcelona, Birkhäuser, Basel 2007, 81-152. Zbl 1188.20026 MR 2281936545

[Sti] J. Stillwell, The occurrence problem for mapping class groups. Proc. Amer. Math. Soc. 101 (1987), no. 3, 411-416. Zbl 0645.20019 MR 0908639538

[Suz] S. Suzuki, On homeomorphisms of a 3-dimensional handlebody. Canad. J. Math. 29 (1977), no. 1, 111-124. Zbl 0339.57001 MR 0433433541

Received January 26, 2009

Nathan Broaddus, Department of Mathematics, University of Chicago, 5734 S. University Ave., Chicago, IL 60637, U.S.A.

E-mail: broaddus@math.uchicago.edu

Benson Farb, Department of Mathematics, University of Chicago, 5734 S. University Ave., Chicago, IL 60637, U.S.A.

E-mail: farb@math.uchicago.edu

Andrew Putman, Department of Mathematics, Rice University, MS 136, 6100 Main St., Houston, TX 77005, U.S.A.

E-mail: andyp@rice.edu 